\title{
Justification and refinement of Winkler-Fuss hypothesis
}

\author{
J. Kaplunov, D. Prikazchikov, L. Sultanova
}

\begin{abstract}
Two-parametric asymptotic analysis of the equilibrium of an elastic half-space coated by a thin soft layer is developed. The initial scaling is motivated by the exact solution of the plane problem for a vertical harmonic load. It is established that the Winkler-Fuss hypothesis is valid only for a sufficiently high contrast in the stiffnesses of the layer and the half-space. As an alternative, a uniformly valid non-local approximation is proposed. Higher order corrections to the Winkler-Fuss formulation, such as the Pasternak model, are also studied.
\end{abstract}

Keywords. Soft thin coating, Asymptotic, Winkler-Fuss model, Contrast, Substrate.

\section{Introduction}

The simplest and apparently the most popular structural model of an elastic foundation is based on the Winkler-Fuss assumption treating a continuum as a system of vertical springs. As mentioned in [20], N. Fuss came to this hypothesis studying a beam floating along the surface of an incompressible fluid, whereas E. Winkler [28] considered a foundation as a mattress consisting of non-connected elastic springs, see also [10] for more detail. It is crucial that, according to the Winkler-Fuss hypothesis, the resulting displacements occur only under the applied force, with the neighbouring area not affected. For further historical remarks on the Winkler-Fuss model the reader is reffered to [13]. A detailed critical review of the Winkler-Fuss, as well as other elastic foundation models, can be found in [16]. Another useful review of foundation models is presented also in [18], taking into account viscoelastic behaviour. One of the focuses of the recent monograph [2] is on the asymptotic analysis of layered and inhomogeneous foundations.

The Winkler-Fuss approximation is widely used in civil engineering $[9,14,21,24,27,29]$ and also in contact mechanics, including, in particular, analysis and interpretation of the experimental results for a depth-sensing indentation, see [5] and references therein. We specially mention its potential in nanoindentation modelling [12]. As a rule, the Winkler-Fuss model may be implemented provided that there is a high contrast in the stiffnesses of a substrate and a coating, when the substrate is much harder. At the same time, for a thin coating supported by an infinite substrate, a limited amount of effort has been made to provide a mathematical justification of this model, see e.g. [3], as well as $[4,19,23]$. These contributions appreciate the general challenge, however, they do not take into consideration a two-parametric nature of the problem, involving geometric and material parameters, expressing relative thickness and stiffness, respectively. The Winkler-Fuss formulation was only fully validated for a layered foundation clamped at the bottom, although without a special emphasis on the contrast properties of the layers, see [2].

This paper is aiming at investigating the area of validity of the Winkler-Fuss model in application to coated solids. A multiparametric asymptotic scheme is adapted for a coated elastic half-space, assuming two small parameters. The first of them is geometrical parameter $\varepsilon$, characteristic of thin plates and coatings $[2,7,11,15]$. Second small parameter, $\mu$, stands for the contrast in stiffnesses of the layer and the half-space. The static behaviour of the coated structure in question is studied 
depending on the value of parameter $\alpha(\alpha \geq 0)$ relating the quantities above by formula $\mu=\varepsilon^{\alpha}$. It is shown that the Winkler-Fuss model is justified only for a rather high contrast, when $\alpha>1$, while for lower contrast $0 \leq \alpha \leq 1$ it fails at leading order. A robust alternative approach valid at $0 \leq \alpha \leq 1$ is proposed starting from the transmission of the prescribed load to the interface between the layer and the half-space. Asymptotic corrections to the Winkler-Fuss hypothesis are also presented over various intervals of parameter $\alpha$.

The paper is organized as follows. The problem is formulated in Section 2 for an elastic coated half-space subject to a vertical load along its surface. The solution of a toy plane problem for a harmonic load is given in Section 3. The asymptotic behaviour of the solution in Section 3 is implemented in Section 4 for the initial scaling underlying a two-parametric approach oriented to justification of the Winkler-Fuss model as a leading order approximation of the related 3D problem. Subsection 4.4 is concerned with the derivation of corrections to this model. The discussion of the obtained results is presented in Section 5. The coefficients in the exact solution in Section 3 are given in Appendix 1. The solution of the plane problem for a homogeneous half-space subject to a harmonic vertical load at its surface is given in Appendix 2.

\section{Statement of the problem}

Consider a linearly isotropic elastic layer $\left(0 \leq x_{3} \leq h\right)$ resting on an elastic half-space $\left(x_{3} \geq h\right)$. Let a vertical force $P=P\left(x_{1}, x_{2}\right)$ be applied at the surface of the layer, see Figure 1 .

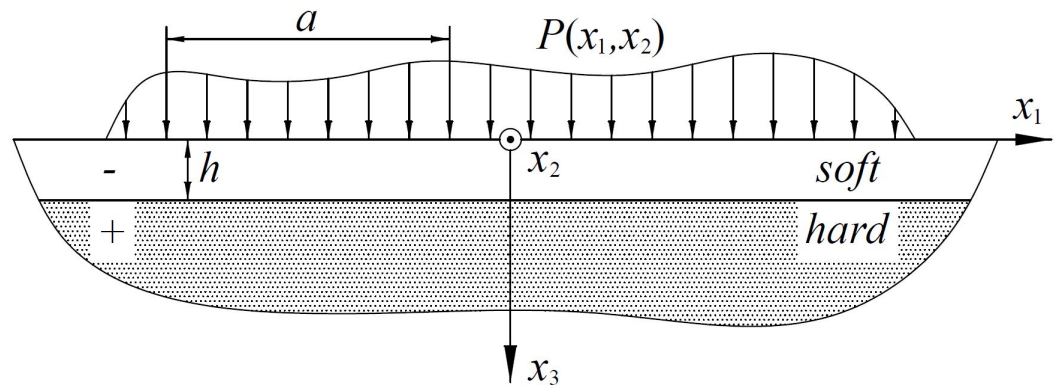

FiguRE 1. Problem statement.

Throughout the paper we assume that the thickness of the layer is small compared to a typical length scale related to the load variation along the coordinates $x_{1}, x_{2}$, and also suppose as a rule that the half-space is much stiffer than the layer.

Let us start from the 3D equations in linear elasticity, given by

$$
\sigma_{i 1,1}^{ \pm}+\sigma_{i 2,2}^{ \pm}+\sigma_{i 3,3}^{ \pm}=0
$$

see e.g. [1], where $\sigma_{i j}$ are Cauchy stresses $(i, j=1,2,3)$. Here and below " -" and "+" correspond to the layer and the half-space, respectively, whereas comma indicates differentiation. The constitutive relations can be written as

$$
\sigma_{i j}^{ \pm}=\lambda^{ \pm} \delta_{i j}\left(u_{1,1}^{ \pm}+u_{2,2}^{ \pm}+u_{3,3}^{ \pm}\right)+\mu^{ \pm}\left(u_{i, j}^{ \pm}+u_{j, i}^{ \pm}\right)
$$

where $u_{i}^{ \pm}$are displacements, $\delta_{i j}$ is Kronecker's delta, $\lambda^{ \pm}$and $\mu^{ \pm}$are Lamé parameters.

The boundary conditions at $x_{3}=0$ modeling vertical loading at the upper face of the layer are

$$
\sigma_{33}^{-}=-P, \quad \sigma_{k 3}^{-}=0,
$$

where $k=1,2$. We also impose the continuity conditions

$$
u_{i}^{-}=u_{i}^{+}, \quad \sigma_{i 3}^{-}=\sigma_{i 3}^{+}
$$

at the interface $x_{3}=h$. 
In view of the assumptions above, we define two small parameters

$$
\varepsilon=\frac{h}{a} \ll 1,
$$

and

$$
\mu=\frac{\mu^{-}}{\mu^{+}} \ll 1
$$

which can be related to each other as

$$
\mu=\varepsilon^{\alpha},
$$

with $\alpha \geq 0$; here the limiting case $\alpha=0$, corresponding to a non-contrast setup, is also included.

On introducing dimensionless variables

$$
\xi_{k}=\frac{x_{k}}{a}, \quad \xi_{3}^{-}=\frac{x_{3}}{h}, \quad 0 \leq x_{3} \leq h, \quad \xi_{3}^{+}=\frac{x_{3}-h}{a}, \quad x_{3} \geq h
$$

we then rearrange basic relations (1) and (2) as

$$
\begin{aligned}
& \sigma_{i 1,1}^{-}+\sigma_{i 2,2}^{-}+\frac{a}{h} \sigma_{i 3,3}^{-}=0, \\
& \sigma_{k k}^{-}=\frac{\lambda^{-}+2 \mu^{-}}{a} u_{k, k}^{-}+\frac{\lambda^{-}}{a} u_{l, l}^{-}+\frac{\lambda^{-}}{h} u_{3,3}^{-}, \\
& \sigma_{33}^{-}=\frac{\lambda^{-}}{a} u_{1,1}^{-}+\frac{\lambda^{-}}{a} u_{2,2}^{-}+\frac{\lambda^{-}+2 \mu^{-}}{h} u_{3,3}^{-}, \\
& \sigma_{12}^{-}=\frac{\mu^{-}}{a}\left(u_{1,2}^{-}+u_{2,1}^{-}\right), \\
& \sigma_{k 3}^{-}=\frac{\mu^{-}}{h}\left(u_{k, 3}^{-}+\frac{h}{a} u_{3, k}^{-}\right),
\end{aligned}
$$

and

with $l, k=1,2 ; l \neq k$.

$$
\begin{aligned}
& \sigma_{i 1,1}^{+}+\sigma_{i 2,2}^{+}+\sigma_{i 3,3}^{+}=0, \\
& \sigma_{k k}^{+}=\frac{1}{a}\left(\left(\lambda^{+}+2 \mu^{+}\right) u_{k, k}^{+}+\lambda^{+} u_{l, l}^{+}+\lambda^{+} u_{3,3}^{+}\right), \\
& \sigma_{33}^{+}=\frac{1}{a}\left(\lambda^{+} u_{1,1}^{+}+\lambda^{+} u_{2,2}^{+}+\left(\lambda^{+}+2 \mu^{+}\right) u_{3,3}^{+}\right), \\
& \sigma_{12}^{+}=\frac{\mu^{+}}{a}\left(u_{1,2}^{+}+u_{2,1}^{+}\right), \\
& \sigma_{k 3}^{+}=\frac{\mu^{+}}{a}\left(u_{k, 3}^{+}+u_{3, k}^{+}\right),
\end{aligned}
$$

For numerous applications the most important consequence of the solution of the stated problem is the relation between the applied load and the vertical displacement of the upper face of the layer. A popular among the engineering community Winkler-Fuss hypothesis [20, 28], suggests that

$$
P=k u_{3}^{-}(0),
$$

where $u_{3}^{-}(0)$ are the displacements of the upper face of the layer and $k$ is a heuristic coefficient.

In this paper we develop a multi-parametric asymptotic procedure, aiming at estimating the range of the parameter $\alpha$, for which the Winkler-Fuss approximation may be mathematically justified. Refinements to this model are also derived in what follows.

\section{Plane problem for a harmonic load}

We begin with a model plane problem for a vertical harmonic force $P=A \mu^{-} \cos \xi_{1}$, see Figure 2 . 


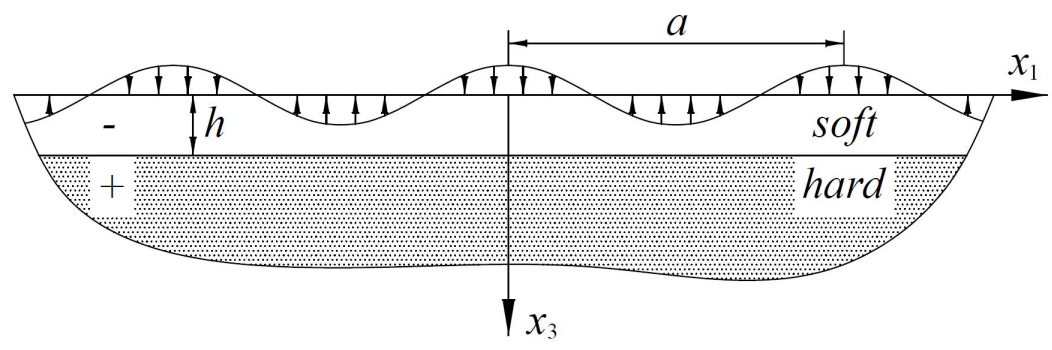

FiguRE 2. Plane problem for a harmonic surface load.

The associated dimensionless equations of equilibrium may be taken in the form of (9) and (10), setting $u_{2}^{ \pm}=0$ and $\frac{\partial}{\partial \xi_{2}}=0$, i.e.

$$
\begin{aligned}
& \left(\lambda^{-}+2 \mu^{-}\right) \varepsilon^{2} u_{1,11}^{-}+\left(\lambda^{-}+\mu^{-}\right) \varepsilon u_{3,13}^{-}+\mu^{-} u_{1,33}^{-}=0, \\
& \mu^{-} \varepsilon^{2} u_{3,11}^{-}+\left(\lambda^{-}+\mu^{-}\right) \varepsilon u_{1,13}^{-}+\left(\lambda^{-}+2 \mu^{-}\right) u_{3,33}^{-}=0, \\
& \left(\lambda^{+}+2 \mu^{+}\right) u_{1,11}^{+}+\left(\lambda^{+}+\mu^{+}\right) u_{3,13}^{+}+\mu^{+} u_{1,33}^{+}=0, \\
& \mu^{+} u_{3,11}^{+}+\left(\lambda^{+}+\mu^{+}\right) u_{1,13}^{+}+\left(\lambda^{+}+2 \mu^{+}\right) u_{3,33}^{+}=0 .
\end{aligned}
$$

The boundary conditions (3) become

$$
\sigma_{33}^{-}=-A \mu^{-} \cos \xi_{1}, \quad \sigma_{13}^{-}=0,
$$

where $A$ is a constant amplitude, with continuity conditions (4). The decay of the spacial displacements is also assumed, i.e. $u_{n}^{+} \rightarrow 0, n=1,3$, as $x_{3} \rightarrow \infty$.

The sought for displacement components may be presented as

$$
u_{1}^{ \pm}=f_{1}^{ \pm}\left(\xi_{3}^{ \pm}\right) \sin \xi_{1}, \quad u_{3}^{ \pm}=f_{3}^{ \pm}\left(\xi_{3}^{ \pm}\right) \cos \xi_{1} .
$$

Then, on substituting (14) into (12), we have

$$
\begin{aligned}
& \mu^{-} f_{1}^{-\prime \prime}\left(\xi_{3}^{-}\right)-\left(\lambda^{-}+2 \mu^{-}\right) \varepsilon^{2} f_{1}^{-}\left(\xi_{3}^{-}\right)-\left(\lambda^{-}+\mu^{-}\right) \varepsilon f_{3}^{-\prime}\left(\xi_{3}^{-}\right)=0, \\
& \left(\lambda^{-}+2 \mu^{-}\right) f_{3}^{-\prime \prime}\left(\xi_{3}^{-}\right)+\left(\lambda^{-}+\mu^{-}\right) \varepsilon f_{1}^{-^{\prime}}\left(\xi_{3}^{-}\right)-\mu^{-} \varepsilon^{2} f_{3}^{-}\left(\xi_{3}^{-}\right)=0, \\
& \mu^{+}{f_{1}^{+\prime \prime}}^{+}\left(\xi_{3}^{+}\right)-\left(\lambda^{+}+2 \mu^{+}\right) f_{1}^{+}\left(\xi_{3}^{+}\right)-\left(\lambda^{+}+\mu^{+}\right){f_{3}^{+}}^{\prime}\left(\xi_{3}^{+}\right)=0, \\
& \left(\lambda^{+}+2 \mu^{+}\right) f_{3}^{+\prime \prime}\left(\xi_{3}^{+}\right)+\left(\lambda^{+}+\mu^{+}\right){f_{1}^{+}}^{+}\left(\xi_{3}^{+}\right)-\mu^{+} f_{3}^{+}\left(\xi_{3}^{+}\right)=0 .
\end{aligned}
$$

The solution of problem (12), (13), and (4) decaying at infinity is given by

$$
\begin{aligned}
& f_{1}^{-}\left(\xi_{3}^{-}\right)=e^{-\varepsilon \xi_{3}^{-}}\left[c_{3}+c_{4}\left(\xi_{3}^{-}-c_{0}^{-}\right)\right]-e^{\varepsilon \xi_{3}^{-}}\left[c_{1}+c_{2}\left(\xi_{3}^{-}+c_{0}^{-}\right)\right], \\
& f_{3}^{-}\left(\xi_{3}^{-}\right)=\left(c_{1}+c_{2} \xi_{3}^{-}\right) e^{\varepsilon \xi_{3}^{-}}+\left(c_{3}+c_{4} \xi_{3}^{-}\right) e^{-\varepsilon \xi_{3}^{-}}, \\
& f_{1}^{+}\left(\xi_{3}^{+}\right)=e^{-\xi_{3}^{+}}\left[c_{5}+c_{6}\left(\xi_{3}^{+}-\varepsilon c_{0}^{+}\right)\right], \\
& f_{3}^{+}\left(\xi_{3}^{+}\right)=\left(c_{5}+c_{6} \xi_{3}^{+}\right) e^{-\xi_{3}^{+}},
\end{aligned}
$$

where the values of $c_{0}^{ \pm}$and $c_{q}, q=1, \ldots, 6$ are given in Appendix 1 . The leading order asymptotic behaviour of the displacements and stresses expressed in terms of small parameter $\varepsilon$ at $\alpha \geq 0$ is given in Table 1.

\begin{tabular}{|c|ccccc|ccccc|}
\hline & $u_{1}^{-}$ & $u_{3}^{-}$ & $\sigma_{13}^{-}$ & $\sigma_{33}^{-}$ & $\sigma_{11}^{-}$ & $u_{1}^{+}$ & $u_{3}^{+}$ & $\sigma_{13}^{+}$ & $\sigma_{33}^{+}$ & $\sigma_{11}^{+}$ \\
\hline$\alpha \geq 2$ & $\varepsilon$ & 1 & $\varepsilon$ & 1 & 1 & $\varepsilon^{\alpha-1}$ & $\varepsilon^{\alpha-1}$ & 1 & 1 & 1 \\
\hline $1 \leq \alpha \leq 2$ & $\varepsilon^{\alpha-1}$ & 1 & $\varepsilon$ & 1 & 1 & $\varepsilon^{\alpha-1}$ & $\varepsilon^{\alpha-1}$ & 1 & 1 & 1 \\
\hline $0 \leq \alpha \leq 1$ & $\varepsilon^{\alpha-1}$ & $\varepsilon^{\alpha-1}$ & $\varepsilon$ & 1 & 1 & $\varepsilon^{\alpha-1}$ & $\varepsilon^{\alpha-1}$ & 1 & 1 & 1 \\
\hline
\end{tabular}

TABLE 1. Asymptotic behaviour of displacements and stresses. 
It is clear that $u_{3}^{-} \gg u_{3}^{+}$at $\alpha \geq 1$, i.e. the deformations of the half-space may be neglected at leading order. Moreover, $u_{3}^{-}$and $\sigma_{33}^{-}$are of the same asymptotic order, which does not contradict the Winkler-Fuss hypothesis. For $0 \leq \alpha \leq 1$ we have $u_{3}^{-} \sim u_{3}^{+}$and also $u_{3}^{-} \ll \sigma_{33}^{-}$, corresponding to a non-negligible effect of the substrate deformability. The value $\alpha=1$ appears to be a boarder line value, since still $u_{3}^{-} \sim \sigma_{33}^{-}$, but at the same time $u_{3}^{-} \sim u_{3}^{+}$.

Now, consider in more detail the relation between vertical displacement $u_{3}^{-}$at upper boundary $\xi_{3}^{-}=0$ and prescribed load $\sigma_{33}^{-}$, see $(13)_{1}$. We have from (14), (16) and (76) (Appendix 1) at leading order

$$
k=\frac{P}{u_{3}^{-}}= \begin{cases}\frac{\lambda^{-}+2 \mu^{-}}{h}, & \alpha>1, \\ \frac{2\left(\gamma^{+}-1\right) \mu^{-}\left(\lambda^{-}+2 \mu^{-}\right)}{h\left[\lambda^{-} \gamma^{+}-2 \mu^{-}\left(1-2 \gamma^{+}\right)\right]}, & \alpha=1, \\ \frac{2\left(\gamma^{+}-1\right) \mu^{-}}{h \gamma^{+}} \varepsilon^{1-\alpha}, & \alpha<1,\end{cases}
$$

where $\gamma^{ \pm}=\frac{\lambda^{ \pm}+2 \mu^{ \pm}}{\mu^{ \pm}}$

Hence, at $\alpha>1$ vertical displacement $u_{3}^{-}$is proportional to prescribed load $P$. In this case, according to Winkler-Fuss hypothesis (11), coefficient $k$ depends only on the thickness and elastic parameters of the layer. This result was earlier obtained in [2] for a layer with a clamped base. At the same time, at $\alpha \leq 1$ this coefficient already depends on both the parameters of the layer and the half-space and can be written down explicitly only for the considered sinusoidal load. It is also worth noting that at $\alpha<1$ factor $\varepsilon^{\alpha-1}$ indicates the violation of Winkler-Fuss type behaviour.

\section{General asymptotic analysis}

Now we are in position to proceed with a more general insight, using the data in Table 1 as a motivation for scaling exploited in the asymptotic procedure below, addressing all of the cases corresponding to the classification in this Table.

\subsection{Case $\alpha \geq 2\left(\mu \lesssim \varepsilon^{2}\right)$}

Let us adapt the asymptotic procedure widely used in mechanics of thin elastic structures, see e.g. $[2,7,11]$. First, scale the displacements and stresses according to the data in the first line of the Table. Then, we have for the layer

$$
\begin{aligned}
& u_{k}^{-}=h \varepsilon u_{k}^{*-}, \quad \sigma_{12}^{-}=\sigma_{21}^{-}=\mu^{-} \varepsilon^{2} \sigma_{12}^{*-}, \quad \sigma_{i i}^{-}=\mu^{-} \sigma_{i i}^{*-}, \\
& u_{3}^{-}=h u_{3}^{*-}, \quad \sigma_{k 3}^{-}=\sigma_{3 k}^{-}=\mu^{-} \varepsilon \sigma_{k 3}^{*-}, \quad P=\mu^{-} p^{*},
\end{aligned}
$$

where the quantities with the asterisk are assumed to be of the same asymptotic order. In view of (18), governing equations (9) may be rewritten as

$$
\begin{aligned}
& \sigma_{k k, k}^{*-}+\varepsilon^{2} \sigma_{12, l}^{*-}+\sigma_{k 3,3}^{*-}=0, \\
& \varepsilon^{2}\left(\sigma_{13,1}^{*-}+\sigma_{23,2}^{*-}\right)+\sigma_{33,3}^{*-}=0, \\
& \sigma_{k k}^{*-}=\varepsilon^{2}\left(\gamma^{-} u_{k, k}^{*-}+\left(\gamma^{-}-2\right) u_{l, l}^{*-}\right)+\left(\gamma^{-}-2\right) u_{3,3}^{*-}, \\
& \sigma_{33}^{*-}=\varepsilon^{2}\left(\gamma^{-}-2\right)\left(u_{1,1}^{*-}+u_{2,2}^{*-}\right)+\gamma^{-} u_{3,3}^{*-}, \\
& \sigma_{12}^{*-}=u_{1,2}^{*-}+u_{2,1}^{*-}, \\
& \sigma_{k 3}^{*-}=u_{k, 3}^{*-}+u_{3, k}^{*-},
\end{aligned}
$$

with comma denoting now differentiation with respect to dimensionless variables $\xi_{i}$.

Here and below the scaling for the half-space is given by

$$
u_{i}^{+}=h \varepsilon^{\alpha-1} u_{i}^{*+}, \quad \sigma_{i j}^{+}=\sigma_{j i}^{+}=\mu^{-} \sigma_{i j}^{*+},
$$


which, on substituting into (10), gives

$$
\begin{aligned}
& \sigma_{i 1,1}^{*+}+\sigma_{i 2,2}^{*+}+\sigma_{i 3,3}^{*+}=0, \\
& \sigma_{k k}^{*+}=\gamma^{+} u_{k, k}^{*+}+\left(\gamma^{+}-2\right)\left(u_{l, l}^{*+}+u_{3,3}^{*+}\right), \\
& \sigma_{33}^{*+}=\gamma^{+} u_{3,3}^{*+}+\left(\gamma^{+}-2\right)\left(u_{1,1}^{*+}+u_{2,2}^{*+}\right), \\
& \sigma_{12}^{*+}=u_{1,2}^{*+}+u_{2,1}^{*+} \\
& \sigma_{k 3}^{*+}=u_{k, 3}^{*+}+u_{3, k}^{*+} .
\end{aligned}
$$

We also have from $(3)$

$$
\sigma_{33}^{*-}=-p^{*}, \quad \sigma_{k 3}^{*-}=0
$$

at $\xi_{3}^{-}=0$, and from $(4)$

$$
u_{k}^{*-}=\varepsilon^{\alpha-2} u_{k}^{*+}, \quad u_{3}^{*-}=\varepsilon^{\alpha-1} u_{3}^{*+}, \quad \varepsilon \sigma_{k 3}^{*-}=\sigma_{k 3}^{*+}, \quad \sigma_{33}^{*-}=\sigma_{33}^{*+}
$$

at $\xi^{-}=1$.

It is clear from $(23)$ that $u_{k}^{*-} \gg u_{k}^{*+}$ at $\alpha>2$ while $u_{k}^{*-} \sim u_{k}^{*+}$ at $\alpha=2$. In what follows we tackle each of these subcases separately.

4.1.1. Subcase $\alpha>2\left(\mu \ll \varepsilon^{2}\right)$. Let us begin with the analysis of the layer by expanding the displacement and stress components in asymptotic series as

$$
\left(\begin{array}{c}
u_{i}^{*-} \\
\sigma_{i j}^{*-}
\end{array}\right)=\left(\begin{array}{c}
u_{i}^{-(0)} \\
\sigma_{i j}^{-(0)}
\end{array}\right)+\varepsilon^{\beta}\left(\begin{array}{l}
u_{i}^{-(1)} \\
\sigma_{i j}^{-(1)}
\end{array}\right)+\ldots,
$$

where

$$
\beta= \begin{cases}2, & \alpha \geq 3, \\ \alpha-1, & 1<\alpha \leq 3 .\end{cases}
$$

At leading order, we have from (19)

$$
\begin{aligned}
& \sigma_{k k, k}^{-(0)}+\sigma_{k 3,3}^{-(0)}=0, \\
& \sigma_{33,3}^{-(0)}=0, \\
& \sigma_{k k}^{-(0)}=\left(\gamma^{-}-2\right) u_{3,3}^{-(0)}, \\
& \sigma_{33}^{-(0)}=\gamma^{-} u_{3,3}^{-(0)}, \\
& \sigma_{12}^{-(0)}=u_{1,2}^{-(0)}+u_{2,1}^{-(0)}, \\
& \sigma_{k 3}^{-(0)}=u_{k, 3}^{-(0)}+u_{3, k}^{-(0)},
\end{aligned}
$$

subject to

$$
\sigma_{33}^{-(0)}=-p^{*}, \quad \sigma_{k 3}^{-(0)}=0,
$$

and

$$
u_{i}^{-(0)}=0, \quad \sigma_{k 3}^{+(0)}=0, \quad \sigma_{33}^{-(0)}=\sigma_{33}^{+(0)}
$$

at $\xi_{3}^{-}=0$ and $\xi_{3}^{-}=1$, respectively.

First, from $(26)_{2}$, satisfying boundary condition $(27)_{1}$, we deduce

$$
\sigma_{33}^{-(0)}=-p^{*} .
$$

Then, $(26)_{4}$ and $(28)_{1}$ imply

$$
u_{3}^{-(0)}=\frac{1}{\gamma^{-}}\left(1-\xi_{3}^{-}\right) p^{*}
$$

which at $\xi_{3}^{-}=0$ validates the Winkler-Fuss hypothesis, see (11) and also the discussion below.

Next, we find the rest of the displacements and stresses. In particular, $(26)_{3}$ yields

$$
\sigma_{k k}^{-(0)}=\frac{2-\gamma^{-}}{\gamma^{-}} p^{*} .
$$


Now, substituting (31) into $(26)_{1}$ and satisfying $(27)_{2}$, we arrive at

$$
\sigma_{k 3}^{-(0)}=\frac{\gamma^{-}-2}{\gamma^{-}} \xi_{3}^{-} \frac{\partial p^{*}}{\partial \xi_{k}} .
$$

Then, using (32), (26) 6 , and (28), we infer

$$
u_{k}^{-(0)}=\frac{1}{2 \gamma^{-}}\left[\left(\gamma^{-}-1\right)\left(\xi_{3}^{-}\right)^{2}-2 \xi_{3}^{-}+3-\gamma^{-}\right] \frac{\partial p^{*}}{\partial \xi_{k}} .
$$

Finally, combining the latter with $(26)_{5}$, we have

$$
\sigma_{12}^{-(0)}=\frac{1}{\gamma^{-}}\left[\left(\gamma^{-}-1\right)\left(\xi_{3}^{-}\right)^{2}-2 \xi_{3}^{-}+3-\gamma^{-}\right] \frac{\partial^{2} p^{*}}{\partial \xi_{1} \partial \xi_{2}} .
$$

Leading order displacement and stress components for the half-space, $u_{i}^{+(0)}$ and $\sigma_{i j}^{+(0)}$, can be expressed through known quantities $u_{i}^{-(0)}$ and $\sigma_{i j}^{-(0)}$. Say, due to continuity condition (28) at $\xi_{3}^{-}=1$, they can be found by substituting given vertical stress in (29), into a convolution with the Boussinesq's solution, e.g. [22].

4.1.2. Subcase $\alpha=2\left(\mu \sim \varepsilon^{2}\right)$. Here we start from same leading order equations (26) and boundary conditions (27) for the layer, while the continuity conditions at $\xi_{3}^{-}=1$ are now given by

$$
u_{k}^{-(0)}=u_{k}^{+(0)}, \quad u_{3}^{-(0)}=0, \quad \sigma_{k 3}^{+(0)}=0, \quad \sigma_{33}^{-(0)}=\sigma_{33}^{+(0)} .
$$

As above, quantities $\sigma_{33}^{-(0)}, u_{3}^{-(0)}, \sigma_{k k}^{-(0)}$ and $\sigma_{k 3}^{-(0)}$ are expressed by (29)-(32), respectively. Then, using (32) and $(26)_{6}$, we deduce

$$
u_{k}^{-(0)}=\frac{1}{2 \gamma^{-}}\left[\left(\gamma^{-}-1\right)\left(\xi_{3}^{-}\right)^{2}-2 \xi_{3}^{+}+3-\gamma^{-}\right] \frac{\partial p^{*}}{\partial \xi_{k}}+\left.u_{k}^{-(0)}\right|_{\xi_{3}^{-}=1} .
$$

Thus, contrary to the subcase $\alpha>2$, the effect of the substrate on horizontal displacements $u_{k}^{-(0)}$ now appears at leading order because of $(35)_{1}$. This is also true for shear stress $\sigma_{12}^{-(0)}$, for which we get from $(26)_{5}$ and $(36)$

$$
\sigma_{12}^{-(0)}=\frac{1}{\gamma^{-}}\left[\left(\gamma^{-}-1\right)\left(\xi_{3}^{-}\right)^{2}-2 \xi_{3}^{+}+3-\gamma^{-}\right] \frac{\partial^{2} p^{*}}{\partial \xi_{1} \partial \xi_{2}}+\left.\left(\frac{\partial u_{1}^{-(0)}}{\partial \xi_{2}}+\frac{\partial u_{2}^{-(0)}}{\partial \xi_{1}}\right)\right|_{\xi_{3}^{-}=1} .
$$
(30).

However, the leading order relation between $u_{3}^{-(0)}$ and $\sigma_{33}^{-(0)}$ is still given by Winkler-Fuss formula

4.2. Case $1 \leq \alpha<2\left(\varepsilon^{2} \lesssim \mu \ll \varepsilon\right)$

The scaling for the layer now takes the form

$$
\begin{aligned}
& u_{k}^{-}=h \varepsilon^{\alpha-1} u_{k}^{*-}, \quad \sigma_{12}^{-}=\sigma_{21}^{-}=\mu^{-} \varepsilon^{\alpha} \sigma_{12}^{*-}, \quad \sigma_{i i}^{-}=\mu^{-} \sigma_{i i}^{*-}, \\
& u_{3}^{-}=h u_{3}^{*-}, \quad \sigma_{k 3}^{-}=\sigma_{3 k}^{-}=\mu^{-} \varepsilon \sigma_{k 3}^{*-}, \quad P=\mu^{-} p^{*} .
\end{aligned}
$$

As a result, governing equations (9) can be written as

$$
\begin{aligned}
& \sigma_{k k, k}^{*-}+\varepsilon^{\alpha} \sigma_{12, l}^{*-}+\sigma_{k 3,3}^{*-}=0, \\
& \varepsilon^{2}\left(\sigma_{13,1}^{*-}+\sigma_{23,2}^{*-}\right)+\sigma_{33,3}^{*-}=0, \\
& \sigma_{k k}^{*-}=\varepsilon^{\alpha}\left(\gamma^{-} u_{k, k}^{*-}+\left(\gamma^{-}-2\right) u_{l, l}^{*-}\right)+\left(\gamma^{-}-2\right) u_{3,3}^{*-}, \\
& \sigma_{33}^{*-}=\varepsilon^{\alpha}\left(\gamma^{-}-2\right)\left(u_{1,1}^{*-}+u_{2,2}^{*-}\right)+\gamma^{-} u_{3,3}^{*-}, \\
& \sigma_{12}^{*-}=u_{1,2}^{*-}+u_{2,1}^{*-}, \\
& \sigma_{k 3}^{*-}=\varepsilon^{\alpha-2} u_{k, 3}^{*-}+u_{3, k}^{*-} .
\end{aligned}
$$

Boundary conditions (22) stay the same, whereas the continuity conditions at $\xi_{3}^{-}=1$ become

$$
u_{k}^{*-}=u_{k}^{*+}, \quad u_{3}^{*-}=\varepsilon^{\alpha-1} u_{3}^{*+}, \quad \varepsilon \sigma_{k 3}^{*-}=\sigma_{k 3}^{*+}, \quad \sigma_{33}^{*-}=\sigma_{33}^{*+} .
$$

Below we consider separately the subcases $1<\alpha<2$ and $\alpha=1$. 
4.2.1. Subcase $1<\alpha<2\left(\varepsilon^{2} \ll \mu \ll \varepsilon\right)$. At leading order, we have for the layer

$$
\begin{aligned}
& \sigma_{k k, k}^{-(0)}+\sigma_{k 3,3}^{-(0)}=0, \\
& \sigma_{33,3}^{-(0)}=0, \\
& \sigma_{k k}^{-(0)}=\left(\gamma^{-}-2\right) u_{3,3}^{-(0)}, \\
& \sigma_{33}^{-(0)}=\gamma^{-} u_{3,3}^{-(0)}, \\
& \sigma_{12}^{-(0)}=u_{1,2}^{-(0)}+u_{2,1}^{-(0)}, \\
& u_{k, 3}^{-(0)}=0,
\end{aligned}
$$

subject to boundary conditions (27) and the following continuity conditions at $\xi_{3}^{-}=1$

$$
u_{k}^{-(0)}=u_{k}^{+(0)}, \quad u_{3}^{-(0)}=0, \quad \sigma_{k 3}^{+(0)}=0, \quad \sigma_{33}^{-(0)}=\sigma_{33}^{+(0)} .
$$

As in subsection 4.1, quantities $\sigma_{33}^{-(0)}, u_{3}^{-(0)}, \sigma_{k k}^{-(0)}$ and $\sigma_{k 3}^{-(0)}$ are given by (29)-(32), respectively. Also, formulae $(41)_{6}$ and $(41)_{5}$ yield

$$
u_{k}^{-(0)}=\left.u_{k}^{-(0)}\right|_{\xi_{3}^{-}=1},
$$

and

$$
\sigma_{12}^{-(0)}=\left.\left(\frac{\partial u_{1}^{-(0)}}{\partial \xi_{2}}+\frac{\partial u_{2}^{-(0)}}{\partial \xi_{1}}\right)\right|_{\xi_{3}^{-}=1}
$$

Here, in contrast to (36) and (37), quantities $u_{k}^{-(0)}$ and $\sigma_{12}^{-(0)}$ in (43) and (44) depend only upon substrate deformations.

4.2.2. Subcase $\alpha=1(\mu \sim \varepsilon)$. Now the leading order equations for the layer are again given by (41), subject to boundary conditions (27), with the continuity conditions at $\xi_{3}^{-}=1$ taking the form

$$
u_{i}^{-(0)}=u_{i}^{+(0)}, \quad \sigma_{k 3}^{+(0)}=0, \quad \sigma_{33}^{-(0)}=\sigma_{33}^{+(0)} .
$$

Remarkably, even though $\alpha=1$, i.e. $\mu=\frac{\mu^{-}}{\mu^{+}} \sim \frac{h}{a}=\varepsilon$, hence $\mu^{-} \ll \mu^{+}$, the half-space is still not stiff enough to behave as an absolutely rigid substrate; compare $(42)_{2}$ and $(45)_{1}$ at $i=3$. Therefore, we cannot expect the validity of the Winkler-Fuss hypothesis.

As before, the quantity of $\sigma_{33}^{-(0)}$ is given by (29), whereas, as it follows from $(41)_{4}$ and $(27)_{1}$,

$$
u_{3}^{-(0)}=\frac{p^{*}}{\gamma^{-}}\left(1-\xi_{3}^{-}\right)+\left.u_{3}^{-(0)}\right|_{\xi_{3}^{-}=1} .
$$

This relation demonstrates that vertical displacement $u_{3}^{-(0)}$ is no longer proportional to prescribed load $p^{*}$ due to presence of an extra term in the right hand side, corresponding to the substrate effect, see $(45)_{1}$ at $i=3$. Thus, the Winkler-Fuss approximation is not valid even at leading order. Also, $(41)_{3},(41)_{1}$ and $(27)_{2}$ imply

$$
\sigma_{k k}^{-(0)}=\frac{\left(2-\gamma^{-}\right)}{\gamma^{-}} p^{*}
$$

and

$$
\sigma_{k 3}^{-(0)}=\frac{\left(\gamma^{-}-2\right)}{\gamma^{-}} \xi_{3}^{-} \frac{\partial p^{*}}{\partial \xi_{k}}
$$

Finally, quantities $u_{k}^{-(0)}$ and $\sigma_{12}^{-(0)}$ now satisfy same formulae (43) and (44), as in the previous subsection, respectively. 
4.3. Case $0 \leq \alpha<1(\varepsilon \lesssim \mu \ll 1)$

The scaling for the layer is

$$
\begin{array}{lll}
u_{i}^{-}=h \varepsilon^{\alpha-1} u_{i}^{*-}, & \sigma_{12}^{-}=\sigma_{21}^{-}=\mu^{-} \varepsilon^{\alpha} \sigma_{12}^{*-}, & P=\mu^{-} p^{*}, \\
\sigma_{i i}^{-}=\mu^{-} \sigma_{i i}^{*-}, & \sigma_{k 3}^{-}=\sigma_{3 k}^{-}=\mu^{-} \varepsilon \sigma_{k 3}^{*-} . &
\end{array}
$$

Consequently, governing equations (9) become

$$
\begin{aligned}
& \sigma_{k k, k}^{*-}+\varepsilon^{\alpha} \sigma_{12, l}^{*-}+\sigma_{k 3,3}^{*-}=0, \\
& \varepsilon^{2} \sigma_{13,1}^{*-}+\varepsilon^{2} \sigma_{23,2}^{*-}+\sigma_{33,3}^{*-}=0, \\
& \varepsilon^{1-\alpha} \sigma_{k k}^{*-}=\varepsilon\left(\gamma^{-} u_{k, k}^{*-}+\left(\gamma^{-}-2\right) u_{l, l}^{*-}\right)+\left(\gamma^{-}-2\right) u_{3,3}^{*-}, \\
& \varepsilon^{1-\alpha} \sigma_{33}^{*-}=\varepsilon\left(\gamma^{-}-2\right)\left(u_{1,1}^{*-}+u_{2,2}^{*-}\right)+\gamma^{-} u_{3,3}^{*-}, \\
& \sigma_{12}^{*-}=u_{1,2}^{*-}+u_{2,1}^{*-} \\
& \varepsilon^{2-\alpha} \sigma_{k 3}^{*-}=u_{k, 3}^{*-}+\varepsilon u_{3, k}^{*-},
\end{aligned}
$$

subject to boundary conditions (22). The continuity conditions at $\xi_{3}^{-}=1$ can be written as

$$
u_{i}^{*-}=u_{i}^{*+}, \quad \varepsilon \sigma_{k 3}^{*-}=\sigma_{k 3}^{*+}, \quad \sigma_{33}^{*-}=\sigma_{33}^{*+} .
$$

At leading order, we get from (50) and (51)

$$
\begin{aligned}
& \sigma_{k k, k}^{-(0)}+\varepsilon^{\alpha} \sigma_{12, l}^{-(0)}+\sigma_{k 3,3}^{-(0)}=0, \\
& \sigma_{33,3}^{-(0)}=0, \\
& u_{i, 3}^{-(0)}=0, \\
& \sigma_{12}^{-(0)}=u_{1,2}^{-(0)}+u_{2,1}^{-(0)},
\end{aligned}
$$

where term $\sigma_{12, l}^{-(0)}$ can be neglected at $0<\alpha<1$, and

$$
u_{i}^{-(0)}=u_{i}^{+(0)}, \quad \sigma_{k 3}^{+(0)}=0, \quad \sigma_{33}^{-(0)}=\sigma_{33}^{+(0)} .
$$

On integrating $(52)_{2}$ and satisfying $(27)_{1}$, we arrive again at $(29)$ for $\sigma_{33}^{-(0)}$. Next, $(52)_{3}$ results in

$$
u_{i}^{-(0)}=\left.u_{i}^{-(0)}\right|_{\xi_{3}^{-}=1} .
$$

All the displacements of the layer are now strongly affected by the presence of the half-space, according to boundary condition $(53)_{1}$.

\subsection{Higher order corrections}

Below, we derive higher order corrections to the relation between vertical displacement of the upper face of the layer $u_{3}^{-}(0)$ and applied load $P$, expressing the studied Winkler-Fuss hypothesis. We restrict ourselves to the range $\alpha>1$, in which it is valid at leading order. The form of asymptotic expansion (24) involving parameter $\beta$, see (25), motivates a separate treatment of cases $\alpha>3, \alpha=3$ and $1<\alpha<3$.

4.4.1. Case $\alpha>3\left(\mu \ll \varepsilon^{3}\right)$. Over this parameter range, the sought for correction is $O\left(\varepsilon^{2}\right)$, see $(24)$ and (25). Then, governing equations $(19)_{2}$ and $(19)_{4}$ become

$$
\begin{aligned}
& \sigma_{13,1}^{-(0)}+\sigma_{23,2}^{-(0)}+\sigma_{33,3}^{-(1)}=0 \\
& \sigma_{33}^{-(1)}=\left(\gamma^{-}-2\right)\left(u_{1,1}^{-(0)}+u_{2,2}^{-(0)}\right)+\gamma^{-} u_{3,3}^{-(1)}
\end{aligned}
$$

subject to the boundary condition at $\xi_{3}^{-}=0$

$$
\sigma_{33}^{-(1)}=0
$$

and the continuity condition at $\xi_{3}^{-}=1$

$$
u_{3}^{-(1)}=0 \text {. }
$$

Thus, at $\alpha>3$ the interface between the layer and the half-space may be treated as a clamped one not only at leading order, but also at the next one. 
Using $(55)_{1},(32)$, and (56), we deduce

$$
\sigma_{33}^{-(1)}=\frac{2-\gamma^{-}}{2 \gamma^{-}}\left(\xi_{3}^{-}\right)^{2} \Delta_{*} p^{*}
$$

where $\Delta_{*}=\frac{\partial^{2}}{\partial \xi_{1}^{2}}+\frac{\partial^{2}}{\partial \xi_{2}^{2}}$ is a $2 \mathrm{D}$ Laplace operator in $\xi_{1}$ and $\xi_{2}$. Then, substituting (33) into $(55)_{2}$, and taking into account $(57)$, we obtain

$$
u_{3}^{(1)}=\frac{\left(2-\gamma^{-}\right)\left(\xi_{3}^{-}-1\right)}{6\left(\gamma^{-}\right)^{2}}\left[6-3 \xi_{3}^{-}+\gamma^{-}\left(\xi_{3}^{-}-2+\left(\xi_{3}^{-}\right)^{2}\right)\right] \Delta_{*} p^{*}
$$

4.4.2. Case $\alpha=3\left(\mu \sim \varepsilon^{3}\right)$. Equations (55) and boundary condition (56) are now complemented by the continuity condition

$$
u_{3}^{-(1)}=u_{3}^{+(0)} .
$$

As a result, we have same expression (58) for $\sigma_{33}^{-(1)}$, whereas $u_{3}^{-(1)}$ becomes

$$
u_{3}^{(1)}=\frac{\left(2-\gamma^{-}\right)\left(\xi_{3}^{-}-1\right)}{6\left(\gamma^{-}\right)^{2}}\left[6-3 \xi_{3}^{-}+\gamma^{-}\left(\xi_{3}^{-}-2+\left(\xi_{3}^{-}\right)^{2}\right)\right] \Delta_{*} p^{*}+\left.u_{3}^{-(1)}\right|_{\xi_{3}^{-}=1},
$$

where the last term in the right hand side is given by (60), and can be found, for example, using the Boussinesq's solution, see [22].

4.4.3. Case $1<\alpha<3\left(\varepsilon^{3} \ll \mu \ll \varepsilon\right)$. In this case the sought for correction is $O\left(\varepsilon^{\alpha-1}\right)$. Therefore,

$$
\begin{aligned}
& \sigma_{33,3}^{-(1)}=0, \\
& \sigma_{33}^{-(1)}=\gamma^{-} u_{3,3}^{-(1)},
\end{aligned}
$$

leading to

and

$$
\sigma_{33}^{-(1)}=0
$$

$$
u_{3}^{-(1)}=\left.u_{3}^{-(1)}\right|_{\xi_{3}^{-}=1} .
$$

Therefore, the next order correction is entirely affected by substrate deformation.

\section{Discussion}

Asymptotic analysis above proves that the Winkler-Fuss hypothesis is valid at leading order at $\alpha>1$ $(\mu \ll \varepsilon)$, see $(30)$ at $\xi_{3}^{-}=0$. In dimensional form it is given by (11) with coefficient $k$ coinciding with its exact value in the first line in (17).

The correction to the Winkler-Fuss model depends upon the value of parameter $\alpha$ over range $\alpha>1$. In particular, at $\alpha>3\left(\mu \ll \varepsilon^{3}\right)$, we have a two-term asymptotic formula, see (30) and (59) at $\xi_{3}^{-}=0$. It is

where $\Delta=\frac{\partial^{2}}{\partial x_{1}^{2}}+\frac{\partial^{2}}{\partial x_{2}^{2}}$ and

$$
u_{3}^{-}(0)=\frac{P}{k}+\frac{T}{k^{2}} \Delta P
$$

$$
T=\frac{h\left(5 \gamma^{-}-\left(\gamma^{-}\right)^{2}-6\right) \mu^{-}}{3} .
$$

Expression (66) for coefficient $\mathrm{T}$ shows that the deformation of the half-space does not influence the calculated correction. It is also clear that the latter is of relative order $O\left(\varepsilon^{2}\right)$. The two-term formula above can also be rewritten in symbolic form as

$$
P=\frac{k u_{3}^{-}(0)}{1+\frac{T}{k} \Delta} \approx k u_{3}^{-}(0)-T \Delta u_{3}^{-}(0),
$$


corresponding to the Pasternak elastic foundation, e.g. see [17, 25, 26].

In case $\alpha=3\left(\mu \sim \varepsilon^{3}\right)$, it might be seen from (30) and (61) that the associated correction involves a term expressing the effect of the half-space, namely

$$
u_{3}^{-}(0)=\frac{P}{k}+\frac{T}{k^{2}} \Delta P+u_{3}^{-}(h),
$$

or again

$$
P \approx k\left(u_{3}^{-}(0)-u_{3}^{-}(h)\right)-T \Delta u_{3}^{-}(0),
$$

where displacements of the interface $\left(x_{3}=h\right) u_{3}^{-}(h)$ is also of order $O\left(\varepsilon^{2}\right)$ according to Table 1 and asymptotic relations $(20)$.

For $1<\alpha<3\left(\varepsilon^{3} \ll \mu \ll \varepsilon\right)$, see (30) and (64), the last term in (68) is greater than the second one, resulting in the two-term asymptotic formula

$$
P=k\left(u_{3}^{-}(0)-u_{3}^{-}(h)\right) .
$$

where $u_{3}^{-}(h)=O\left(\varepsilon^{\alpha-1}\right)$. Displacement $u_{3}^{-}(h)$ in $(68)$ - (70), due to continuity condition $(4)_{1}$ at $i=3$, may be found from a simpler problem for a homogeneous half-space $x_{3} \geq h$ with boundary conditions $\sigma_{33}^{+}=-P$ and $\sigma_{k 3}^{+}=0$, see also (28) and (29) for more detail. For a harmonic load $P=A \mu^{-} \cos \xi_{1}$, its solution is given in Appendix 2.

As it has already been mentioned, the Winkler-Fuss hypothesis fails at leading order at $0 \leq \alpha \leq 1$ $(\varepsilon \ll \mu \lesssim 1)$. In particular, it is violated even at $\mu=\frac{\mu^{-}}{\mu^{+}} \sim \varepsilon=\frac{h}{a}$, which is still a relatively large contrast, as it follows from (46) at $\alpha=1$, and also confirmed by comparison with the exact solution, see the second line in (17).

Formula (70) can be also written as

$$
P=k \delta u_{3}^{-},
$$

where $\delta u_{3}^{-}=u_{3}^{-}(0)-u_{3}^{-}(h)$. It is worth noting that outside the range of validity of the Winkler-Fuss hypothesis at $\alpha=1$, the leading order solution will also take the form of $(71)$, in which $u_{3}^{-}(0) \sim$ $u_{3}^{-}(h) \sim k P$, see $(46)$ at $\xi_{3}^{-}=0$.

Finally, we remark that at $0 \leq \alpha<1$ (71) becomes

$$
\delta u_{3}^{-}=0,
$$

reflecting almost uniform variation of the transverse displacements across the thickness of the layer. It is obvious that this approximation is also valid at non-contrast limit $\alpha=0$. Moreover, interfacial values $u_{3}^{-}(h)$ at $0 \leq \alpha \leq 1$ may be found at leading order from the the problem for a homogeneous half-space as in formulae (68) - (70) above.

Next, we test the validity of the Winkler-Fuss hypothesis and its asymptotic corrections, by comparing them with the exact solution of the plane problem for harmonic load $P=A \mu^{-} \cos \xi_{1}$ applied at upper face $x_{3}=0$. We study the normalized coefficient

$$
k_{*}=\frac{h P}{\mu^{-} u_{3}^{-}(0)}
$$

using formulae above; in doing so, interfacial displacements $u_{3}^{-}(h)$ in (68) - (72) are given by (82), see Appendix 2. In this case

$$
k_{*}= \begin{cases}\gamma^{-}, & \text {formula (11) with } k \text { from the first line in (17), } \\ \frac{3 l^{2}\left(\gamma^{-}\right)^{2}}{3 l^{2} \gamma^{-}-h^{2}\left(5 \gamma^{-}-\left(\gamma^{-}\right)^{2}-6\right),} & \text { formula (65), } \\ \frac{2 h \gamma^{-}\left(\gamma^{+}-1\right)}{l \gamma^{-} \gamma^{+} \mu+2 h\left(\gamma^{+}-1\right),} & \text { formula (70), } \\ \frac{2 h\left(\gamma^{+}-1\right)}{l \gamma^{+} \mu,} & \text { formula (72). }\end{cases}
$$




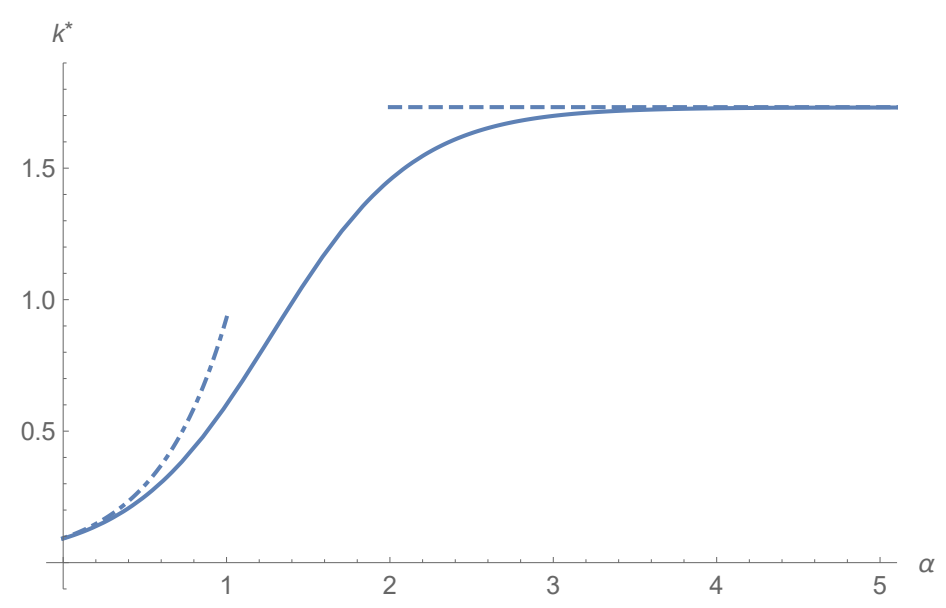

Figure 3. Approximate and exact solutions for harmonic load

The related exact solution takes the form

$$
k_{*}=\frac{A h}{c_{1}+c_{3}}
$$

with constants $c_{1}$ and $c_{3}$ given by (76), see Appendix 1 .

Numerical results are presented in Figure 3, in which $\alpha=\log _{\varepsilon} \mu$ and also $\nu^{-}=0.25, \nu^{+}=0.3$, and $\varepsilon=h / l=0.1$. The exact solution is plotted with solid line, while the graphs corresponding to the Winkler-Fuss hypothesis (the first line in (74)) and the formula in the forth line in (74) based on the assumption of uniform variation of the transverse displacements across the thickness of the layer, are displayed by dashed and dot-dashed lines, respectively. The Figure shows that two aforementioned formulae have limited ranges of applicability. At the same time, the formula in the third line of (74) appears to be uniformly valid, and the associated curve in the Figure can not be distinguished from that for the exact solution. We also mention that the deviation of the straight line corresponding to the Pasternak model, see the second line in (74), from that for the Winkler-Fuss one is only $0.07 \%$.

\section{Conclusion}

A two-parametric asymptotic approach, operating with two small parameters, is developed. It is established that only at a rather high contrast, when $\mu \ll \varepsilon$, and substrate deformations are negligible, the Winkler-Fuss hypothesis is validated. It does however unexpectedly fail at $\mu \sim \varepsilon$, when the relative thickness and stiffness of the coating are of the same order. It is also shown that the prescribed vertical surface load may always be transmitted to the interface. As a result, the sought for interfacial displacements follow from a simpler problem for a homogeneous half-space, leading to uniform twoterm asymptotic formula (70) valid over the whole range of the contrast parameter. It is worth noting that along with local Winkler-Fuss term $k u_{3}^{-}(0)$, this formula contains non-local term $k u_{3}^{-}(h)$, see also [6] and [8], addressing current trends in modelling of nonlocal elastic foundations. In general case the term $k u_{3}^{-}(0)$ is given by a convolution using Boussinesq's solution [22]. Higher order corrections to the Winkler-Fuss approximation are also derived, including that corresponding to the Pasternak model, see e.g. $[17,25,26]$. 


\section{Appendix 1}

The constants in (16) are

$$
c_{0}^{ \pm}=\frac{1}{\varepsilon}\left(1+\frac{2 \mu^{ \pm}}{\lambda^{ \pm}+\mu^{ \pm}}\right), \quad c_{q}=\frac{N_{q}}{Z}, \quad q=1, \ldots, 6,
$$

where

$$
\begin{aligned}
N_{1}= & A h\left(2 \mathrm{e}^{2 \varepsilon} \varepsilon^{2} B_{1}^{-} B_{2}^{+}+\left(\gamma^{-}\right)^{2}\left[\mathrm{e}^{2 \varepsilon}\left(B_{8}^{+}+\varepsilon^{2 \alpha}+2 \varepsilon^{1+\alpha} B_{10} B_{12}^{+}+\gamma^{+} B_{1}^{-} B_{1}^{+} B_{8}^{+}\right)-B_{1}^{-} B_{3}\right]\right. \\
& \left.-\gamma^{-}\left(\mathrm{e}^{2 \varepsilon}\left(\varepsilon^{2 \alpha}-B_{6}^{+}+2 \varepsilon^{1+\alpha} B_{5}^{+} B_{10}+\gamma^{+}\left[B_{6}^{+}+\varepsilon^{2 \alpha}\left(2 \varepsilon B_{5}^{+}+1\right)\right]\right)-B_{1}^{-} B_{2}^{+}\right)\right), \\
N_{2}= & -A h \varepsilon B_{1}^{-} B_{4}^{-}\left(B_{2}^{+}+\mathrm{e}^{2 \varepsilon} B_{2}^{+} B_{4}^{-} B_{5}^{+}-\gamma^{-} B_{3}\right), \\
N_{3}= & A h \mathrm{e}^{2 \varepsilon}\left(\left(\gamma^{-}\right)^{2}\left(2 \varepsilon^{1+\alpha} B_{10} B_{12}^{-}-B_{8}^{-}-\varepsilon^{2 \alpha}+\mathrm{e}^{2 \varepsilon} B_{1}^{+} B_{2}^{+}-\gamma^{+} B_{1}^{-} B_{1}^{+} B_{8}^{-}\right)-2 \varepsilon^{2} B_{1}^{-} B_{2}^{+}\right. \\
& \left.+\gamma^{-}\left(\varepsilon^{2 \alpha}-B_{6}^{-}+2 \varepsilon^{1+\alpha} B_{5}^{-} B_{10}-\mathrm{e}^{2 \varepsilon} B_{1}^{-} B_{2}^{+}+\gamma^{+}\left(B_{6}^{-}+\varepsilon^{2 \alpha}\left(1+2 \varepsilon B_{5}^{-}\right)\right)\right)\right), \\
N_{4}= & A h \mathrm{e}^{2 \varepsilon} \varepsilon B_{2}^{+} B_{4}^{-}\left(B_{1}^{-} B_{4}^{-} B_{5}^{-}+\mathrm{e}^{2 \varepsilon} B_{2}^{-}\right), \\
N_{5}= & 2 A h \mathrm{e}^{\varepsilon} \gamma^{-} \varepsilon^{\alpha}\left(\gamma^{+} \varepsilon+B_{12}^{-}\left(B_{9}-1\right)+\gamma^{-}\left[B_{1}^{-}\left(\varepsilon-\gamma^{+} B_{12}^{-}\right)-\varepsilon^{\alpha}\right]+\right. \\
& \left.\mathrm{e}^{2 \varepsilon}\left[1-\varepsilon B_{4}^{+}-B_{9} B_{12}^{+}+\gamma^{-}\left(\varepsilon B_{1}^{-}+\varepsilon^{\alpha}+\gamma^{+} B_{1}^{+} B_{12}^{+}\right)\right]\right), \\
N_{6}= & 2 A h \mathrm{e}^{\varepsilon} \gamma^{-} B_{4}^{+} \varepsilon^{\alpha}\left(B_{1}^{-} B_{4}^{-} B_{5}^{-}+\mathrm{e}^{2 \varepsilon} B_{2}^{-}\right),
\end{aligned}
$$

and

$$
\begin{aligned}
Z= & 2 \varepsilon\left[B_{2}^{-} B_{2}^{+} B_{4}^{-} \mathrm{e}^{4 \varepsilon}+B_{1}^{-} B_{4}^{-}\left(B_{3} \gamma^{-}-B_{2}^{+}\right)-2 \mathrm{e}^{2 \varepsilon}\left(B_{1}^{-} B_{7} B_{2}^{+}+2 \gamma^{-}\left(2 B_{4}^{+} \varepsilon^{2}\right.\right.\right. \\
& \left.\left.\left.+\varepsilon^{\alpha}\left(1+4 \varepsilon^{2}-B_{7} B_{9}\right)\right)+\left(\gamma^{-}\right)^{2}\left(B_{7}\left(1+B_{1}^{-} B_{1}^{+} \gamma^{+}+\varepsilon^{2 \alpha}\right)-4 \varepsilon^{2+\alpha}\right)\right)\right]
\end{aligned}
$$

with

$$
\begin{array}{lll}
B_{1}^{ \pm}=\varepsilon^{\alpha} \pm 1, & B_{5}^{ \pm}=2 \varepsilon \pm 1, & B_{9}=\left(\gamma^{+}+1\right) \varepsilon^{\alpha}, \\
B_{2}^{ \pm}=\gamma^{ \pm} B_{1}^{+} \pm B_{1}^{-}, & B_{6}^{ \pm}=1-2 \varepsilon B_{5}^{ \pm}, & B_{10}=\varepsilon^{\alpha}-2, \\
B_{3}=B_{1}^{+}+\gamma^{+} B_{1}^{-}, & B_{7}=1+2 \varepsilon^{2}, & B_{11}=\gamma^{+}-3, \\
B_{4}^{ \pm}=\gamma^{ \pm}-1, & B_{8}^{ \pm}=B_{7} \pm 2 \varepsilon & B_{12}^{ \pm}=1 \pm \varepsilon .
\end{array}
$$

\section{Appendix 2}

Consider a plane strain problem for an elastic half-space $x_{3} \geq h$ starting from equations (12) $)_{3}$ and $(12)_{4}$. Let a vertical harmonic load $P=A \mu^{-} \cos \xi_{1}$ be applied at its upper face, corresponding to the boundary conditions

$$
\sigma_{33}^{+}=-A \mu^{-} \cos \xi_{1}, \quad \sigma_{k 3}^{+}=0 .
$$

The solution of the formulated problem is given by $(16)_{3}$ and $(16)_{4}$ with the values of $c_{0}^{+}, c_{5}$ and $c_{6}$, corresponding to boundary conditions (80), expressed as

$$
c_{0}^{+}=\frac{1}{\varepsilon}\left(1+\frac{2 \mu^{+}}{\lambda^{+}+\mu^{+}}\right), \quad c_{5}=\frac{A l \gamma^{+} \mu^{-}}{2 \mu^{+}\left(\gamma^{+}-1\right)}, \quad c_{6}=\frac{A l \mu^{-}}{2 \mu^{+}} .
$$

Then, the displacement at the upper face $\xi_{3}^{+}=0$ becomes

$$
u_{3}^{+}=\frac{A l \gamma^{+} \mu \cos \xi_{1}}{2\left(\gamma^{+}-1\right)},
$$

with $\mu$ defined in (6).

\section{References}

[1] Achenbach, J.: Wave propagation in elastic solids. Elsevier (2012)

[2] Aghalovyan, L.: Asymptotic theory of anisotropic plates and shells. World Scientific (2015)

[3] Aleksandrova, G.P.: Contact problems in bending of a slab lying on an elastic foundation. Izv. Akad. Nauk SSSR, Mekh. Tverd. Tela (1) (1973)

[4] Alexandrov, V.M.: Contact problems on soft and rigid coatings of an elastic half-plane. Mech. solids 45(1), 34-40 (2010)

[5] Borodich, F.M.: The Hertz-type and adhesive contact problems for depth-sensing indentation. Adv. Appl. Mech. 47, 225-366 (2014) 
[6] Challamel, N., Meftah, S.A., Bernard, F.: Buckling of elastic beams on non-local foundation: A revisiting of Reissner model. Mech. Res. Commun. 37(5), 472-475 (2010)

[7] Dai, H.-H., Kaplunov, J., Prikazchikov, D.A.: A long-wave model for the surface elastic wave in a coated half-space. Proc. R. Soc. A 466(2122), 3097-3116 (2010)

[8] Di Paola, M., Marino, F., Zingales, M.: A generalized model of elastic foundation based on long-range interactions: Integral and fractional model. Int. J. Solids Struct. 45(17), 3124-3137 (2009)

[9] Dutta, S.C., Roy, R.: A critical review on idealization and modeling for interaction among soil-foundationstructure system. Comput. struct. 80(20-21), 1579-1594 (2002)

[10] Epshtein, S.A., Borodich, F.M., Bull, S.J.: Evaluation of elastic modulus and hardness of highly inhomogeneous materials by nanoindentation. Appl. Phys. A 119(1), 325-335 (2015)

[11] Erbaş, B., Yusufoğlu, E., Kaplunov, J.: A plane contact problem for an elastic orthotropic strip. J. Eng. Math. 70(4), 399-409 (2011)

[12] Fischer-Cripps, A.C.: Nanoindentation testing. Nanoindentation, Springer, 21-37 (2011)

[13] Frýba, L.: History of Winkler foundation. Veh. Syst. Dyn. 24(1), 7-12 (1995)

[14] Gazetas, G., Dobry, R.: Horizontal response of piles in layered soils. J. Geotech. eng. 110(1), 20-40 (1984)

[15] Goldenveizer, A.L., Kaplunov, J.D., Nolde, E.V.: On Timoshenko-Reissner type theories of plates and shells. Int. J. Solids Struct. 30(5), 675-694 (1993)

[16] Hetenyi, M.: Beams on Elastic Foundation. The University of Michigan Press, Ann Arbor (1958)

[17] Kaplunov, J., Nobili, A.: The edge waves on a Kirchhoff plate bilaterally supported by a two-parameter elastic foundation. J. Vib. Control 23(12), 2014-2022 (2017)

[18] Kerr, A.D.: Elastic and viscoelastic foundation models. J. Appl. Mech. 31(3), 491-498 (1964)

[19] Kovalenko, E.V., Buyanovskii, I.A.: On the durability prediction for an elastic cylinder-elastic layer pair reinforced by thin coating according to the wear criterion. J. Mach. Manuf. Reliab. 44(4), 357-362 (2015)

[20] Kuznetsov, V.I.: Elastic Foundations. Gosstroiizdat, Moscow (1952)

[21] Liyanapathirana, D.S., Poulos, H.G.: Pseudostatic approach for seismic analysis of piles in liquefying soil. J. Geotech. Geoenvironmental Eng. 131(12), 1480-1487 (2005)

[22] Love, A.E.H.: A treatise on the mathematical theory of elasticity. Cambridge university press (2013)

[23] Martynyak, R.M., Prokopyshyn, I.A., Prokopyshyn, I.I.: Contact of Elastic Bodies with Nonlinear Winkler Surface Layers. J. Math. Sci. 205(4), 535-553 (2015)

[24] Nikolaou, S., Mylonakis, G., Gazetas, G., Tazoh, T.: Kinematic pile bending during earthquakes: analysis and field measurements. Geotechnique 51(5), 425-440 (2001)

[25] Nobili, A.: Superposition principle for the tensionless contact of a beam resting on a Winkler or a Pasternak foundation. J. Eng. Mech. 139(10), 1470-1478 (2012)

[26] Pasternak, P.L.: On a new method of an elastic foundation by means of two foundation constants. Gos. Izd. Lit. po Stroit. i Arkhitekture (1954)

[27] Wang, Y.H., Tham, L.G., Cheung, Y.K.: Beams and plates on elastic foundations: a review. Prog. Struct. Eng. Mater. 7(4), 174-182 (2005)

[28] Winkler, E.: Die Lehre von der Elastizit at und Festigkeit. Domimicus, Prague (1867)

[29] Yim, C.-S., Chopra, A.K.: Earthquake response of structures with partial uplift on Winkler foundation. Earthq. eng. struct. dyn. 12(2), 263-281 (1984)

J. Kaplunov, D. Prikazchikov, L. Sultanova

School of Computing and Mathematics, Keele University, Keele, Staffordshire, ST55BG, UK

e-mail: j.kaplunov@keele.ac.uk, d.prikazchikov@keele.ac.uk, 1.sultanova@keele.ac.uk 